\title{
Registration of Micro-PET and High Resolution MR Images of Mice for Monitoring Photodynamic Therapy
}

\author{
Baowei Fei ${ }^{\text {a*}}$, Raymond F. Muzic ${ }^{\text {b,a }}$, Zhenghong Lee ${ }^{\text {b,a }}$, Chris A. Flask ${ }^{\text {b,a }}$, \\ Rachel L. Morris ${ }^{c}$, Jeffery L. Duerk ${ }^{b, a}$, Nancy Oleinick ${ }^{c}$, David L. Wilson ${ }^{a b * *}$ \\ ${ }^{a}$ Department of Biomedical Engineering, Case Western Reserve University, OH 44106 \\ ${ }^{\mathrm{b}}$ Department of Radiology, University Hospitals of Cleveland, OH 44106 \\ ${ }^{c}$ Department of Radiation Oncology, Case Western Reserve University, OH 44106
}

\begin{abstract}
We are investigating imaging techniques to study the rapid biochemical and physiological response of tumors to photodynamic therapy (PDT). Positron emission tomography (PET) can provide physiological and functional images of cancers. While MRI can provide high resolution anatomical images and generate serial, noninvasive, in vivo observations of morphological changes. In this study, we investigate image registration methods to combine MRI and micro-PET ( $\mu$ PET) images for improved tumor monitoring. We acquired high resolution MR and PET ${ }^{18} \mathrm{~F}$ fluorodeoxyglucose (FDG) images from mice with RIF-1 tumors. We used rigid body registration with three translations and three angular variables. We used normalized mutual information as the similarity measure. To assess the quality of registration, we performed slice by slice review of both image volumes, manually segmented feature organs such as the left and right kidneys and the bladder in each slice, and computed the distance between corresponding centroids of the organs. We also used visual inspection techniques such as color overlay displays. Over 40 volume registration experiments were performed with MR and $\mu \mathrm{PET}$ images acquired from three $\mathrm{C} 3 \mathrm{H}$ mice. The color overlays showed that the MR images and the PET images matched well. The distance between corresponding centroids of organs was $1.5 \pm 0.4 \mathrm{~mm}$ which is about 2 pixels of $\mu$ PET. In conclusion, registration of high resolution MR and $\mu$ PET images of mice may be useful to combine anatomical and functional information that could be used for the potential application in photodynamic therapy.
\end{abstract}

Keywords: Image registration, mutual information, magnetic resonance imaging (MRI), positron emission tomography (PET), photodynamic therapy (PDT), micro-PET ( $\mu$ PET), cancers.

\section{INTRODUCTION}

Photodynamic therapy (PDT) is USFDA approved (with the photosensitizer Photofrin) for advanced esophageal, early lung and late lung cancer. ${ }^{1,2}$ In PDT, a tumor-localized photosensitizer is irradiated with visible light to generate reactive oxygen that efficiently kills cells and ablates tumors. PDT is a three-component treatment. ${ }^{1,2}$ It requires (a) a photosensitizer, often a porphyrin-related macrocycle, that tends to accumulate in tumors; (b) non-thermal visible light

* Baowei.Fei@case.edu, **dlw@po.cwru.edu, Wickenden Building 319, 10900 Euclid Avenue, Cleveland, OH 44106.

Medical Imaging 2004: Physiology, Function, and Structure from Medical Images, edited by Amir A. Amini, Armando Manduca, Proceedings of SPIE Vol. 5369

(SPIE, Bellingham, WA, 2004). 1605-7422/04/\$15.00 . doi: 10.1117/12.535465 
of a wavelength absorbed by the photosensitizer and generally in the red region of the spectrum (since longer wavelengths penetrate human tissue best); and (c) molecular oxygen. With an adequate oxygen supply and light intensity, the site of photodamage depends on the location of the photosensitizer. An important advantage of PDT is that both the photosensitizer and the light are inert by themselves, and the light can be precisely focused onto a selected region, allowing extreme specificity in the localization of the photodynamic effect. Consequently, systemic toxicities are minimized.

Imaging techniques are used for the study of cancer. Positron emission tomography (PET) is widely used to study the physiology of many types of cancer. ${ }^{3,4}$ Since tissue uptake of ${ }^{18} \mathrm{~F}$-fluorodeoxyglucose (FDG) depends on both blood flow and hexokinase activity, it can be a good probe to investigate the mechanisms of PDT that dependent on the two parameters. However, because FDG uptake depends on both, it is difficult to differentiate on the basis of FDG alone. We had planned to also do water (perfusion) and carbon monoxide (blood volume). Lapointe et al. reported the study of PET FDG imaging for monitoring the response of tumors to PDT. ${ }^{3}$ A micro-PET ( $\mu$ PET) imager was designed and built to perform dynamic in vivo PET scans of tumor-bearing mice after PDT. Reductions in FDG uptake of a treated tumor relative to an untreated tumor in the same animal were observed. ${ }^{3}$ Magnetic resonance imaging (MRI) has also been used to evaluate PDT-induced hemorrhagic necrosis in the murine M1 tumor within 72 hours of treatment of male DBA/2 mice. ${ }^{5-7}$

In this project, we investigate using both $\mu \mathrm{PET}$ and high-resolution MR imaging techniques for improved tumor monitoring. Though PET images can provide the dynamic functional information, MR has superior anatomical information for the locations of tumors and organs and can make serial, noninvasive, in vivo observations of morphological changes. Conversely, PET can provide functional information that is not available in normal anatomical MR images. The goal of this project is to apply PET imaging to study the rapid biochemical and physiological response of tumors to PDT and to use MR to monitor the anatomical and morphological changes of tumors.

The combination of $\mu$ PET and high-resolution MRI has several advantages. First, MRI scans provide anatomical reference to the PET images. Second, registration of PET and MRI images can enhance our ability to visualize the location of the FDG uptake. Third, MRI provides tumor shape and size information that can be used to improve the accuracy of the PET data analysis such as drawing region of interests (ROIs) and performing quantitative analyses. Fourth, MRI can be used to correct PET data for partial volume effects to clarify that the PET-measured changes induced by PDT are due to metabolic and hemodynamic changes and not artifacts due to changes in tumor size.

In this study, we focus on image registration methods for the alignment of MRI and $\mu$ PET images. Several reports described the registration of MRI and PET for the cat brain, ${ }^{8}$ the rat brain, ${ }^{9}$ a brain phantom, ${ }^{10}$ and radiotherapy planning. ${ }^{11}$ There is no report on mutual information image registration of $\mu$ PET and MRI for the study of photodynamic therapy. We acquired both PET and MR images from mice with tumors and performed over 40 registration experiments. Evaluation results from visual inspection and quantitative measurements are reported. 


\section{REGISTRATION ALGORITHM}

\subsection{Similarity Measurements}

Based on our previous experience, ${ }^{12,13}$ we chose normalized mutual information (NMI) as the similarity measure in our registration because it is robust and suitable for multi-modality image registration. ${ }^{14,15}$ One image $R$ is the reference, and the other $F$ is floating. Their normalized mutual information (NMI) is given by the following equation. ${ }^{15}$

$$
N M I(R, F)=\frac{2 M I(R, F)}{H(R)+H(F)}
$$

where

$$
\begin{aligned}
& H(R)=-\sum_{r} p_{R}(r) \log p_{R}(r) \\
& H(F)=-\sum_{f} p_{F}(f) \log p_{F}(f) \\
& M I(R, F)=\sum_{r, f} p_{R F}(r, f) \log \frac{p_{R F}(r, f)}{p_{R}(r) \cdot p_{F}(f)} .
\end{aligned}
$$

The joint probability $p_{R F}(r, f)$ and the marginal probabilities $p_{R}(r)$ of the reference image and $p_{F}(f)$ of the floating image, can be estimated from the normalized joint intensity histograms. When two images are geometrically aligned, NMI is maximal. ${ }^{15}$

\subsection{Registration Algorithm}

The input data include the PET transmission, emission, and high-resolution MR images. We normally discretize the image intensity to 256 levels. We combine the PET transmission and emission images and form one data set by taking a weighted sum. We assume that there is no movement between the transmission and emission scans. We use the combined PET data and the high-resolution MR image for mutual information registration.

We used rigid body transformation (three translations and three rotations) and trilinear interpolation as described previously. ${ }^{16}$ For optimization, we used the downhill simplex method of Nelder and Mead. ${ }^{17}$ Optimization of similarity ends either when the maximum number (800) of calculations is reached or the fractional change in similarity function is smaller than a tolerance (0.001). Typically the latter is achieved within about 200 iterations. Our very first initial guess is all zeros for the 3 displacements and 3 angles.

\section{EXPERIMENTAL METHODS}

\subsection{Animal Preparation}

RIF (Radiation-induced fibrosarcoma)-1 cells were grown as monolayers in E-MEM supplemented with 15\% fetal bovine serum (ref. 18). Prior to inoculation, $\mathrm{C} 3 \mathrm{H} / \mathrm{HeN}$ mice were shaved and depilated. Two tumors were initiated in 
each mouse by injection of $10^{5}-10^{6}$ RIF-1 cells intradermally on the shoulder flanks, as far from the bladder and kidneys as possible to minimize spillover in PET images. Tumors were treated and imaged when they reach 3-5 mm in diameter, which required 7-10 days after implantation. Animals were given the photosensitizer Pc 4 (1 mg/kg) by tail vein injection. After 24 hours, one of the tumors was exposed to red light $(670 \mathrm{~nm})$ from a diode laser $\left(150 \mathrm{~J} / \mathrm{cm}^{2}\right.$; $\left.150 \mathrm{~mW} / \mathrm{cm}^{2}\right)$.

Three animals were studied for $\mu \mathrm{PET}$ and MR imaging. We know from experience that neither the light nor the photosensitizer alone produces any response, so initially, one of the two tumors in each animal served as a control (receiving photosensitizer but no light), and the other tumor was exposed to laser light.

\subsection{MR Image Acquisition}

Two days after photosensitizer injection, the animals were taken to the MR imaging facility. The mouse images were acquired using a Siemens Sonata 1.5 T scanner (Siemens Medical Systems, Erlangen, Germany). A custom-designed whole-body mouse coil (2-element phased-array, ID $=32 \mathrm{~mm}$ ) was used to minimize noise levels. A T1-weighted spin echo pulse sequence (TR/TE $=600 / 13 \mathrm{~ms}$ ) with a slice thickness of 1-mm was used to generate high-resolution coronal images (Matrix $=256 \times 120, \mathrm{FOV}=80 \times 36-\mathrm{mm}$ ). The number of signal averages was typically set at six to obtain low noise images. In these T1 images, the tumors are clearly delineated by the bright subcutaneous fat signal. During each imaging session, the animals were mounted on a plastic holder and were provided with a continuous supply of $2 \%$ isoflurane (EZAnesthesia, Palmer, PA) in oxygen to minimize motion artifacts in MR images.

\subsection{Micro-PET Image Acquisition}

After MR image acquisition, the animals with the plastic holder and the lasers were taken to the PET imaging facility. We used a MicroPET R4 scanner (Concorde Microsystems, Inc., Knoxville, TN 37932) designed specifically for imaging small rodents. ${ }^{20}$ The in vivo functional imaging with microPET ${ }^{\circledR}$ allows both serial and longitudinal studies to be conducted in the same animal. We followed a single animal over 90-minute period of time and monitored the response of PDT and the outcome.

We used ${ }^{18}$ F-FDG (fludeoxyglucose) that is the standard radiopharmaceutical used in PET scanning for tumor diagnosis and assessment, for cerebral glucose metabolism, and for myocardial metabolic assessment. It has become a standard commodity and is obtained commercially as well as being produced on the premises. It was produced for this experiment in standard fashion. ${ }^{21,22}$

The control tumor was shielded with black cloth, and the animals were placed in plastic holders to restrain them during imaging. The mouse was studied for FDG accumulation, blood flow and blood volume. Depending upon the simulations, ${ }^{18} \mathrm{~F}-\mathrm{FDG}$ was injected as a bolus or by continuous infusion into the tail vein. About 6 min later, laser light was focused onto a 1-cm spot encompassing the non-shielded tumor. FDG accumulation in both tumors was measured during the 15-min light exposure.

We acquired both transmission and emission images from the same mouse. Since the animal did not move during the image session, we assume the there is no movement between the two scans. The PET images include 63 transverse slices covering the whole mouse. Each slice has 128x128-pixel with an in-plane pixel size of $0.85 \mathrm{x} 0.85$-mm and a thickness of $1.2 \mathrm{~mm}$. 


\subsection{Preprocessing}

The input MR volume is a 2D MR acquisition giving $256 \times 120$ with an in-plane resolution of $0.3 \times 0.3-\mathrm{mm}$ and a slice thickness of $1.0-\mathrm{mm}$. Twenty nine coronal slices over a field of view cover the whole mouse. Using tri-linear interpolation, we create isotropic voxels of $0.3 \mathrm{~mm}$ on a side for both PET and MR image volumes. We optionally discretize the intensity to 256 levels. We use IDL (Interactive Data Language, Research System Inc., Boulder, CO) as the programming language.

For purposes of registration, we optionally cropped image slices that were not of interest. For example, the tumors were on the mouse back near the shoulder, we cropped out images at the abdomen and tail. Before cropping, the image volume was $350 \times 250 \times 250$-voxel covering the whole mouse. After cropping, we created a volume with 128x128x248voxel near the region of interest. Cropping processing can bring two advantages for the mouse registration. First, cropping out regions that are not of interest can increase image consistency for the mutual information registration. Since the mouse body is very flexible, the deformation at the abdomen can cause inconsistency for the rigid body registration. Second, the small number of voxels after cropping can increase the speed of image registration.
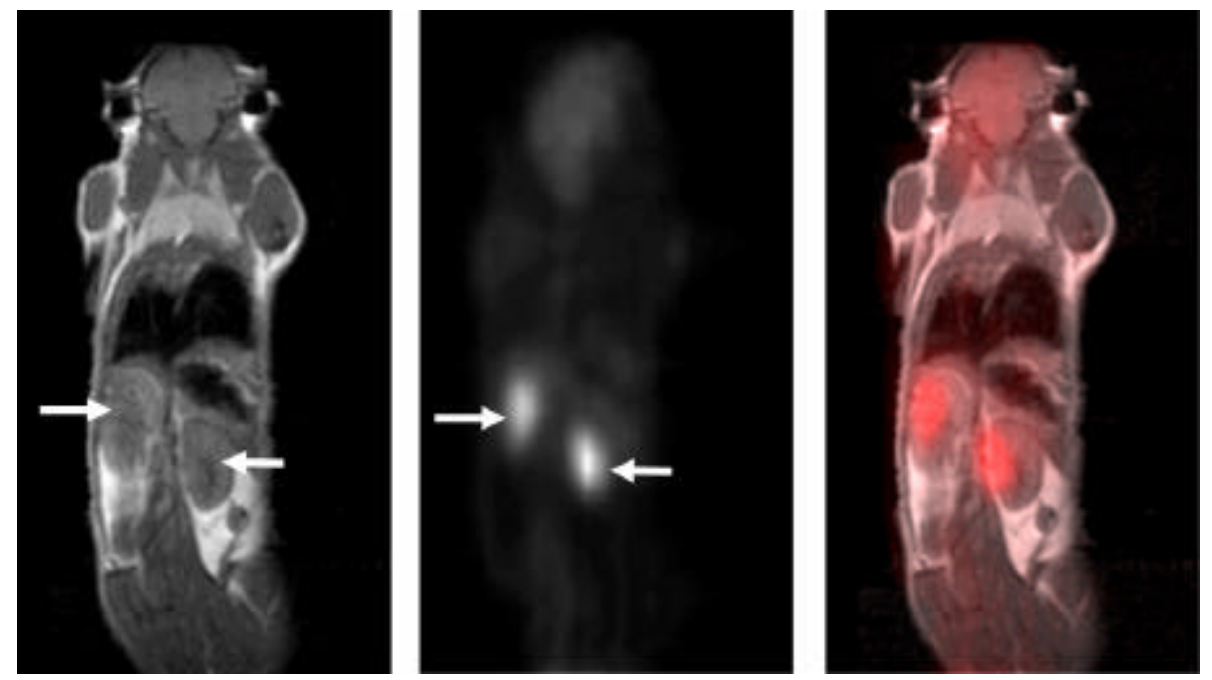

Figure 1. Visual inspection of the kidney registration. Image on the left is the high-resolution MR image. The white arrows indicate the left and right kidneys of the mouse. Image at the center is the PET FDG image.The white arrows indicate the kidneys. Image on the right is the overlay of the two images, which indicate good registration at the organs. Images are from the Mouse No.3. 

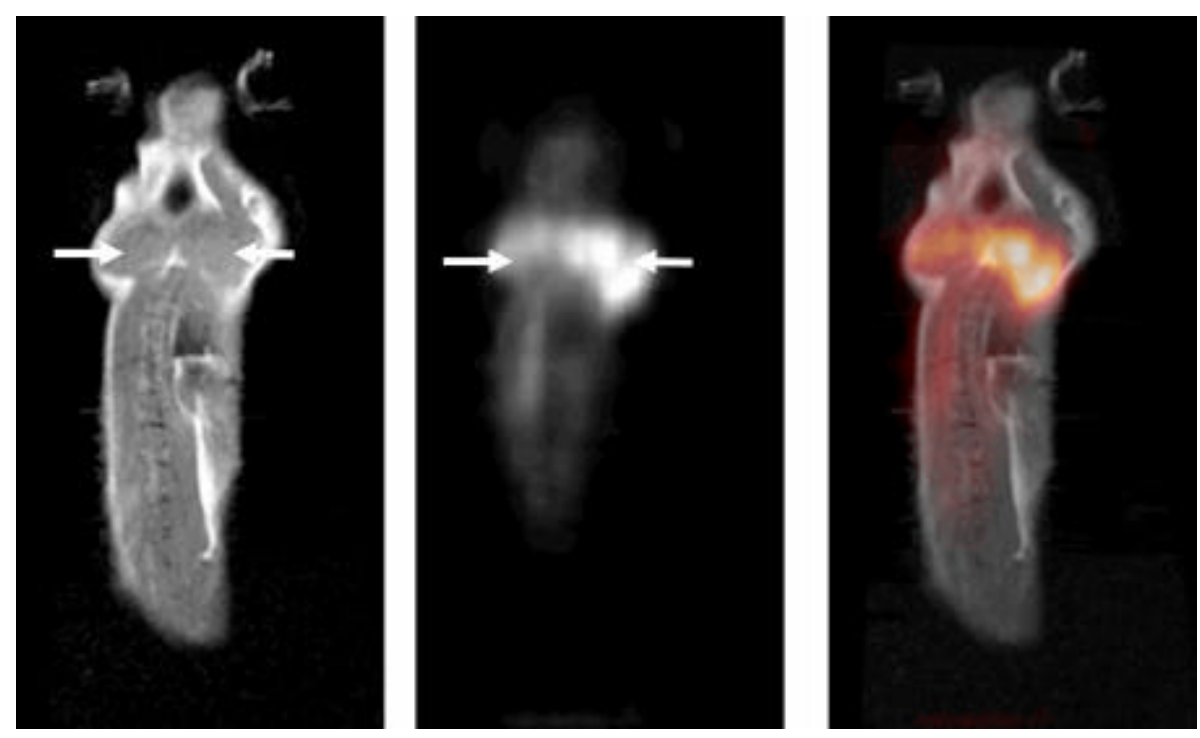

Figure 2. Registration of tumors. Image on the left is the coronal MR image. The white arrows indicate two tumors on the back of the mouse. Image at the center is the PET FDG image. The white arrows indicate two tumors. Image on the right is the overlay image. The tumors are registered well. Images are from the Mouse No.3.

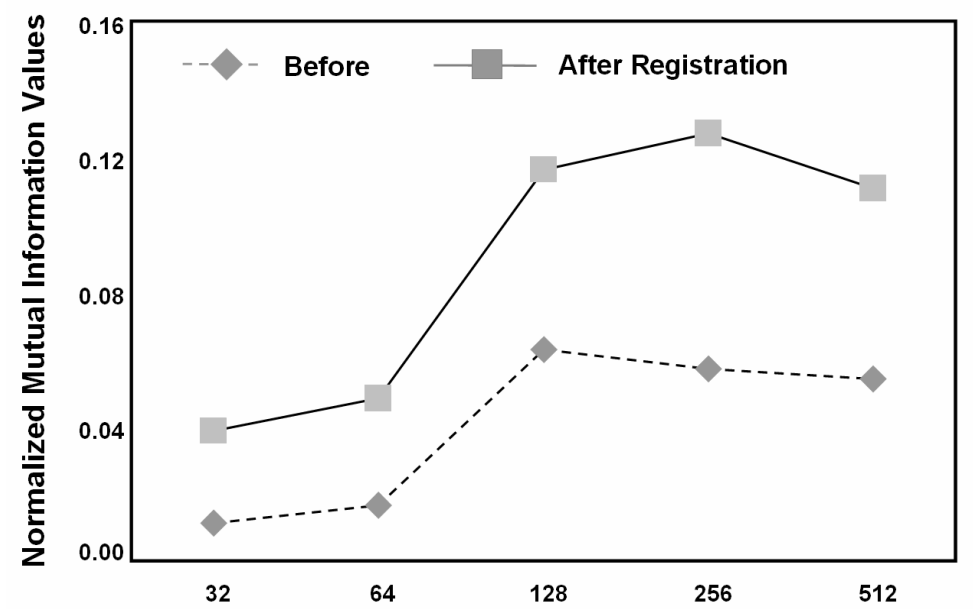

Bins for both MR and PET Transmission Images

Figure 3. The effect of intensity scaling on image registration. The X-axis is the gray levels from 32 to 512. The Y-axis it the normalized mutual information values between MR and PET transmission images. The dash line indicates the NMI values before registration. The real line is the NMI values after registration. 


\section{Results and Discussion}

\subsection{Visual Inspection}

Figure 1 shows the registration of the left and right kidneys of a mouse. The MR image provides the shape of the kidneys. The PET image shows the FDG uptake of the organs. The fused image combines both anatomic and functional information. We also examined other slices in different positions. The kidneys were well-aligned in three dimensions. The kidneys are good anatomic markers for registration evaluation. Other feature organs such as the bladder and heart were also aligned.

Figure 2 shows the registration of two tumors. The high resolution MR image contains the anatomic structure of the tumors. The PET image shows the radioactivity of the tumors. The overlay image indicates good registration of the tumors. We also used other visualization methods found in MIM ${ }^{\mathrm{TM}}$ (MIMvista Corp., Cleveland, Ohio 44122) for the image display and analysis.

\subsection{Effects of Intensity Scaling}

We tested the effect of intensity scaling on image registration. Since PET images are stored as float data, we normally discretize the data to 256 gray levels for image display and processing. We then use the scaled data and apply the mutual information algorithm. Different intensity scaling creates different joint histograms that affect the mutual information calculation. We performed registration experiments using different intensity scaling such as 512, 256, 128,64 , or 32 bins for both volume data sets. Scaling was linear between zero and the maximum value. Registration was examined by analyzing the normalized mutual information values and visual inspection.

In Figure 3, we show the NMI values between the PET transmission image and MR image volume. First, the NMI values increased after registration. Second, when the bin size is 256 , the NMI is the maxim indicating the gray level of 256 is the best for the registration. The visual inspection confirmed the results.

\subsection{Quantitative Evaluation}

We used features identifiable in both PET and MR images to evaluate the mouse registration. We manually segmented feature organs such as the left and right kidneys and the bladder in each slice for both MR and PET image volumes. We calculated the area and the center point for each slice. Then, we compute the three-dimensional centroid of the organs. To evaluate the registration error, we computed the distance between corresponding centroids. For three mice, the registration error is $1.5 \pm 0.4 \mathrm{~mm}$, about $2 \mu \mathrm{PET}$ pixels. More importantly, $\mu \mathrm{PET}$ resolution is about $2 \mathrm{~mm} .^{20}$

\subsection{Combining PET Emission and Transmission Images}

We found that only using PET emission image led to the registration failure and that it is important to add transmission image for the registration. We think the anatomic information from transmission image may be good for the mutual information image registration. However, increasing the percentage of transmission image from $10 \%$ to $100 \%$ made no significant difference. 


\section{CONCLUSION}

We created a rigid body image registration method for $\mu \mathrm{PET}$ and high MR images for a new application in small animal imaging. The registration and fusion provided both functional and anatomic information that could be useful for the potential application in photodynamic therapy. We are going to perform more imaging experiments and we are developing deformable registration method for small animals.

\section{ACKNOWLEDGEMENTS}

The algorithm developed in this research was supported by NIH grant P20 In Vivo Cellular and Molecular Imaging Center to Case Western Reserve University and the DOD grant DAMD17-02-1-0230 to Baowei Fei.

\section{REFERENCE}

1 T.J.Dougherty, "An update on photodynamic therapy applications," J Clin.Laser Med Surg., vol. 20, pp. 3-7, Feb, 2002.

2 T.J.Dougherty, C.J.Gomer, B.W.Henderson, G.Jori, D.Kessel, M.Korbelik, J.Moan, and Q.Peng, "Photodynamic therapy," J Natl.Cancer Inst., vol. 90, pp. 889-905, Jun 17, 1998.

3 D.Lapointe, N.Brasseur, J.Cadorette, C.La Madeleine, S.Rodrigue, J.E.van Lier, and R.Lecomte, "Highresolution PET imaging for in vivo monitoring of tumor response after photodynamic therapy in mice," $J$ Nucl.Med, vol. 40, pp. 876-882, May, 1999.

4 J.V.Moore, M.L.Waller, S.Zhao, N.J.Dodd, P.D.Acton, A.P.Jeavons, and D.L.Hastings, "Feasibility of imaging photodynamic injury to tumours by high-resolution positron emission tomography," Eur.J Nucl.Med, vol. 25, pp. 1248-1254, Sep, 1998.

5 B.G.Winsborrow, H.Grondey, H.Savoie, C.A.Fyfe, and D.Dolphin, "Magnetic resonance imaging evaluation of photodynamic therapy-induced hemorrhagic necrosis in the murine M1 tumor model," Photochem.Photobiol., vol. 66, pp. 847-852, Dec, 1997.

6 S.Gross, A.Gilead, A.Scherz, M.Neeman, and Y.Salomon, "Monitoring photodynamic therapy of solid tumors online by BOLD-contrast MRI," Nat.Med, vol. 9, pp. 1327-1331, Oct, 2003.

7 S.D.Kennedy, L.S.Szczepaniak, S.L.Gibson, R.Hilf, T.H.Foster, and R.G.Bryant, "Quantitative MRI of GdDTPA uptake in tumors: response to photodynamic therapy," Magn Reson Med, vol. 31, pp. 292-301, Mar, 1994.

8 Y.Shimada, K.Uemura, B.A.Ardekani, T.Nagaoka, K.Ishiwata, H.Toyama, K.Ono, and M.Senda, "Application of PET-MRI registration techniques to cat brain imaging," J Neurosci.Methods, vol. 101, pp. 1-7, Aug 15, 2000 .

9 N.Hayakawa, K.Uemura, K.Ishiwata, Y.Shimada, N.Ogi, T.Nagaoka, H.Toyama, K.Oda, A.Tanaka, K.Endo, and M.Senda, "A PET-MRI registration technique for PET studies of the rat brain," Nucl.Med Biol., vol. 27, pp. 121-125, Feb, 2000.

10 M.Koole, Y.D'Asseler, K.Van Laere, W.R.Van de, W.C.Van de, I.Lemahieu, and R.A.Dierckx, "MRI-SPET and SPET-SPET brain co-registration: evaluation of the performance of eight different algorithms," Nucl.Med Commun., vol. 20, pp. 659-669, Jul, 1999.

11 S.J.Kiebel, J.Ashburner, J.B.Poline, and K.J.Friston, "MRI and PET coregistration--a cross validation of statistical parametric mapping and automated image registration," Neuroimage., vol. 5, pp. 271-279, May, 1997. 
12 B.W.Fei, A.Wheaton, Z.Lee, J.L.Duerk, and D.L.Wilson, "Automatic MR volume registration and its evaluation for the pelvis and prostate," Physics in Medicine and Biology, vol. 47, pp. 823-838, 2002.

13 B.W.Fei, J.L.Duerk, D.T.Boll, J.S.Lewin, and D.L.Wilson, "Slice to volume registration and its potential application to interventional MRI guided radiofrequency thermal ablation of prostate cancer," IEEE Transactions on Medical Imaging, vol. 22, pp. 515-525, 2003.

14 A.Collignon, F.Maes, D.Delaere, D.Vandermeulen, P.Suetens, and G.Marchal, "Automated multimodality image registration using information theory,"Information Processing in Medical Imaging: Proc. 14th International Conference (IPMI'95), Computational Imaging and Vision,pp. 287-298, 1995.

15 F.Maes, A.Collignon, D.Vandermeulen, G.Marchal, and P.Suetens, "Multimodality image registration by maximization of mutual information," IEEE Transactions on Medical Imaging, vol. 16, pp. 187-198, 1997.

16 B.W.Fei, Z.Lee, J.L.Duerk, and D.L.Wilson, "Image Registration for Interventional MRI Guided Procedures: Similarity Measurements, Interpolation Methods, and Applications to the Prostate," Lecture Notes of Computer Science (LNCS), vol. 2717, pp. 3292003.

17 J.Nelder and R.A.Mead, "A simplex method for function minimization," Computer Journal, vol. 7, pp.308313, 1965.

18 S.I.Zaidi, N.L.Oleinick, M.T.Zaim, and H.Mukhtar, "Apoptosis during photodynamic therapy-induced ablation of RIF-1 tumors in $\mathrm{C} 3 \mathrm{H}$ mice: electron microscopic, histopathologic and biochemical evidence," Photochem.Photobiol., vol. 58, pp. 771-776, Dec, 1993.

19 C.H.Sibata, V.C.Colussi, N.L.Oleinick, and T.J.Kinsella, "Photodynamic therapy in oncology," Expert. Opin. Pharmacother., vol. 2, pp. 917-927, Jun, 2001.

20 C.Knoess, S.Siegel, A.Smith, D.Newport, N.Richerzhagen, A.Winkeler, A.Jacobs, R.N.Goble, R.Graf, K.Wienhard, and W.D.Heiss, "Performance evaluation of the microPET R4 PET scanner for rodents," Eur.J Nucl.Med Mol. Imaging, vol. 30, pp. 737-747, May, 2003.

21 R.F.Muzic, Jr., B.Landmeier, Z.Zhang, L.Zheng, and M.S.Berridge, "Solid-phase analysis method for (S)[18F]fluorocarazolol and its metabolites," J Chromatogr.B Biomed Sci.Appl., vol. 759, pp. 355-359, Aug 15, 2001.

22 R.F.Muzic, Jr. and S.Cornelius, "COMKAT: compartment model kinetic analysis tool," $J$ Nucl.Med, vol. 42, pp. 636-645, Apr, 2001. 
Baowei Fei, Raymond F. Muzic, Zhenghong Lee, Chris A. Flask, Rachel L. Morris, Jeffery L. Duerk, Nancy Oleinick, and David L. Wilson, "Registration of micro-PET and high-resolution MR images of mice for monitoring photodynamic therapy", Amir A. Amini, Armando Manduca, Proc. SPIE 5369, 371 (2004)

Copyright 2004 Society of Photo-Optical Instrumentation Engineers (SPIE). One print or electronic copy may be made for personal use only. Systematic reproduction and distribution, duplication of any material in this paper for a fee or for commercial purposes, or modification of the content of the paper are prohibited.

http://dx.doi.org/10.1117/12.535465 